\title{
COMPLETE AUTOMORPHISM GROUPS
}

\author{
BY JOAN L. DYER AND EDWARD FORMANEK ${ }^{1}$ \\ Communicated by Hyman Bass, October 31, 1974
}

Let $A(G)$ denote the automorphism group of the group $G$. If the center of $G$ is trivial, the action of $G$ on itself by conjugation identifies $G$ with the group of inner automorphisms, which is a normal subgroup of $A(G)$. Since $A(G)$ is again centerless, this gives rise to an automorphism tower

$$
G \triangleleft A(G) \triangleleft A(A(G))=A^{2}(G) \triangleleft \cdots
$$

which may continue transfinitely. A group is termed complete if it is centerless and if every automorphism is inner. Thus the automorphism tower of a centerless group $G$ is finite if and only if some $A^{r}(G)$ is complete. Burnside [2, p. 95] had observed that $A(G)$ is complete if and only if $G$ is a characteristic subgroup; that is, if and only if $G$ is normal in $A^{2}(G)$. He also established $[2$, p. 96] that a centerless simple group has a complete automorphism group. It was shown by Wielandt [14] that every finite group with trivial center has a finite automorphism tower. In contrast to these results, Hulse [8] proved that the automorphism tower of a centerless polycyclic-and therefore linear-group need not terminate after finitely many steps, although the tower is in this case at most countable.

The work to be described here was motivated by Gilbert Baumslag's conjecture that a sufficiently symmetric group was likely to have a very short tower, and specifically that this would be the case for a free group. We established

THEOREM $1[3] . A(F)$ is complete if $F$ is a free group of finite rank $n \geqslant 2$.

This confirms Baumslag's conjecture in the sharpest sense: the automorphism tower of a free group stabilizes in precisely one step. Theorem 1 is rather special, in that we had available considerable information about $A(F)$,

AMS (MOS) subject classifications (1970). Primary 20F55, 20E25; Secondary 20E05, 20E15, $20 \mathrm{~F} 15$.

1 The second author was supported by NSF Grant GP-36418X1. 
due principally to Nielsen [12] and Magnus [9] (or see [11, pp. 162-169]), as well as the fact that the torsion-free nilpotent images of $F$ distinguish conjugates (Baumslag-Higman [1]). However, we are able to prove

THEOREM 2 [4]. Suppose $R$ is a characteristic proper subgroup of $F$, such that $F / R$ is residually torsion-free nilpotent. Then $A\left(F / R^{\prime}\right)$ is complete.

For groups of the form $F / R^{\prime}$, results of Magnus [10] and Fox [5] (or see [13]) enable us to translate statements about $F / R^{\prime}$ into statements about the integral group ring $\mathrm{Z}[F / R]$. This proved the basic tool used in establishing Theorem 2. The following results, which seem of independent interest, were also obtained. They may be regarded as extensions to $F / R^{\prime}$ of properties possessed by $F$. Theorem 3 extends the Baumslag-Higman theorem mentioned above, and Theorem 4 extends a result of Grossman [6]. In the following, a primitive element of $F / R^{\prime}$ is the image of a primitive element of $F$.

Theorem 3 [4]. Suppose $R \leqslant F^{\prime}$ and $F / R$ is residually torsion-free nilpotent. If $x$ is a primitive element of $F / R^{\prime}$, then the conjugates of $x$ are distinguished in the torsion-free nilpotent images of $F / R^{\prime}$.

THEOREM 4 [4]. Suppose $R \leqslant F^{\prime}, F / R$ is torsion-free, and $\alpha \in A\left(F / R^{\prime}\right)$. If $\alpha(x)$ and $x$ are conjugate in $F / R^{\prime}$ for each primitive element $x$ of $F / R^{\prime}$, then $\alpha$ is inner.

We remark that the residual torsion-free nilpotence of $F / R$ implies that of $F / R^{\prime}$; this latter result is a special case of a theorem due to Hartley [7]. Theorem 3 does not assert that the nilpotent images of $F / R^{\prime}$ distinguish conjugates, and this is in fact false.

Finally, we record here one consequence of a theorem in [4] :

THEOREM 5 [4]. Suppose $C$ is a proper characteristic subgroup of $F$ such that $G=F / C$ is centerless and residually torsion-free nilpotent. Set $K=\operatorname{Ker}\left\{A(G) \rightarrow A\left(G / G^{\prime}\right)\right\}$. If $K / G$ is centerless and residually nilpotent, then $A(G)$ is complete.

Complete proofs and related results will appear elsewhere.

\section{REFERENCES}

1. G. Baumslag, Residual nilpotence and relations in free groups, J. Algebra 2 (1965), 271-282. MR 31 \#3487.

2. W. S. Burnside, Theory of groups of finite order, 2nd ed. Dover, 1955. MR 16, 1086. 
3. J. L. Dyer and E. Formanek, The automorphism group of a free group is complete, Proc. London Math. Soc. (to appear). mitted).

4. - Characteristic subgroups and complete automorphism groups (sub-

5. R. H. Fox, Free differential calculus. I: Derivation in the free group ring, Ann. of Math (2) 57 (1953), 547-560. MR 14, 843.

6. E. K. Grossman, On the residual finiteness of certain mapping class groups, J. London Math. Soc. (to appear).

7. B. Hartley, The residual nilpotence of wreath products, Proc. London Math. Soc. (3) 20 (1970), 365-392. MR 41 \#3611.

8. J. A. Hulse, Automorphism towers of polycyclic groups, J. Algebra 16 (1970), 347-398. MR $42 \# 1888$. 353-367.

9. W. Magnus, Über n-dimensionale Gittertransformationen, Acta Math. 64 (1935),

10. On a theorem of Marshall Hall, Ann. of Math. 40 (1939), 764-768. MR 1, 44.

11. W. Magnus, A. Karrass and D. Solitar, Combinatorial group theory: Presentations of groups in terms of generators and relations, Pure and Appl. Math., vol. 13, Interscience, New York, 1966. MR 34 \#7617.

12. J. Nielsen, Die Isomorphismengruppe der freien Gruppen, Math. Ann. 91 (1924), 169-209.

13. V. N. Remeslennikov and V. G. Sokolov, Certain properties of the Magnus embedding, Algebra i Logika 9 (1970), 566-578 = Algebra and Logic 9 (1970), 342-349. MR 45 \#2001.

14. H. Wielandt, Eine Verallgemeinerung der invariante Untergruppen, Math. $\mathrm{Z}$. 45 (1939), 209-244.

DEPARTMENT OF MATHEMATICS, LEHMAN COLLEGE, CITY UNIVERSITY OF NEW YORK, BRONX, NEW YORK 10468

ISTITUTO MATEMATICA, UNIVERSITÀ DI PISA, VIA DERNA 1, 56100 PISA, ITALY 